\title{
INFLUENCE OF FREON HEAT TREATMENT ON OPTICAL PROPERTIES OF Er-DOPED SILICA-GEL GLASSES
}

\author{
G.E. Malasimkevich \\ Institute of Molecular and Atomic Spectroscopy, Academy of Sciences of Belarus \\ Minsk, Belarus
}

E.N. Poddenezirny, I.M. Melnichenko, V.E. Gaishun, A.V. Semchenko

F. Skarina Gomel State University, Gomel, Belarus

K. Maruszewski and W. StręK

Institute for Low Temperalure and Structure Researcl, Polish Academy of Sciences

Okólna 2, 50-950 Wroclaw, Poland

Theeffect of freon heat treatment on optical properties of Er-doped silica glasses obtained by the sol-gel method was investigated. It was found that fluorine ions in the process of hydrolysis replace hydroxyl ions in the host and form the complexes with $\mathrm{Er}^{3+}$ ions. It leads to a significant improvement of optical properties of Er doped silica glass.

PACS numbers: 78.55.IIx

\section{Introduction}

A significant interest in preparation and investigation of optical properties of Er-doped silica glasses has been recently noticed. Such glasses are important in telecommunication systems as materials for construction of signal amplifiers [1]. The glasses may be obtained by the sol-gel technology which allows us to produce high optical quality silica glasses at low temperatures [2]. The most essential limitation of the sol-gel glass is a ligh concentration of OII- groups which are the principal technological factor decreasing the luminescence quantum yield of Er-doped silica glasses. A significant decrease in the hydroxyl ion concentration is possible by thermal treatment of xerogels in a freon atmosphere [1]. In the present work we report the influence of such treatment on optical characteristics of Er-doped silica glasses obtained via the sol-gel method. 


\section{Experimental}

The samples of monolithic xerogels were made according to procedures previously described by us [3], by applying an impregnation method for co-doping xerogels using water solutions of $\mathrm{ErCl}_{3} \cdot 6 \mathrm{II}_{2} \mathrm{O}$. The activated xerogels were then heated in a freon $\left(\mathrm{C}_{2} \mathrm{Cl}_{3} \mathrm{~F}_{3}\right)$ atmosphere at $1000^{\circ} \mathrm{C}$ for 2 hours. Then the samples were vitrified in the air at $1250^{\circ} \mathrm{C}$ for two hours. All the reagents used were analytically pure.

The absorption spectra were measured on a Beckman-UV 5270 spectrophotometer and luminescence on a SDL-2 spectrofuorimeter. All measurements were performed at room temperature. The recorded luminescence spectra were corrected taking into account the spectral sensitivity of the recording system. They are presented as the wavelength dependence of luminescence quantum number per unit interval of wavelength, $\mathrm{d} n(\lambda) / \mathrm{d} \lambda$.

\section{Results}

Figure 1 shows the light attenuation spectra of freon heat treated Er-doped silica glass samples obtained by the sol-gel method. The nominal concentrations of $\mathrm{Er}$ ions $C_{\mathrm{Er}}$ (mass \%) for the investigated samples were equal to 0.25 (trace 1), 1 (trace 2) and 3 (trace 3). For comparative purposes we obtained the spectrum

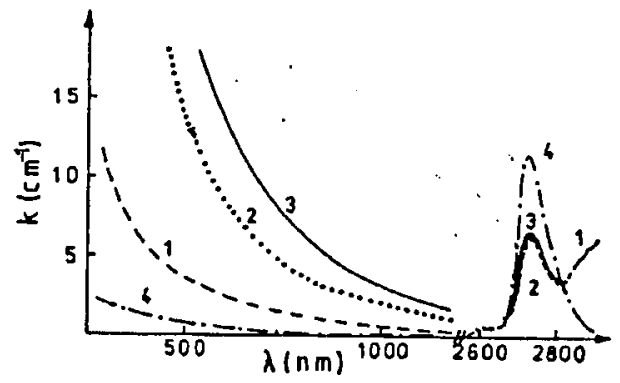

Fig. 1. Light attenuation spectra of Er-doped silica sol-gel glasses treated in freon atmosphere before vitrification (traces 1-3) and non-treated samples (trace 4).

of the sample without the freon treatment (the concentration was 0.5 mass \% (trace 4)). From this figure it can be seen that the concentration of hydroxyl ions decreased several times (the absorption coefficient decreases almost three orders of magnitude in the region of hydroxyl ion fundamental oscillations (2720 nm [4]). The concentration of the hydroxyl impurity was observed to be almost independent of Er concentration, whereas for the freon heat-treated samples we observed a significant increase in the light scattering with increasing erbium concentration $\left(C_{\mathrm{Er}}\right)$.

Figure 2 presents the normalized Er luminescence spectra of ${ }^{4} I_{13 / 2} \rightarrow{ }^{4} I_{15 / 2}$ band of the freon heated Er-doped silica glass samples obtained by the sol-gel method. It should be noted that the intensities of the long-wavelength component of the luminescence band (at $1550 \mathrm{~mm}$ ) of the freon heated samples (1-3) 


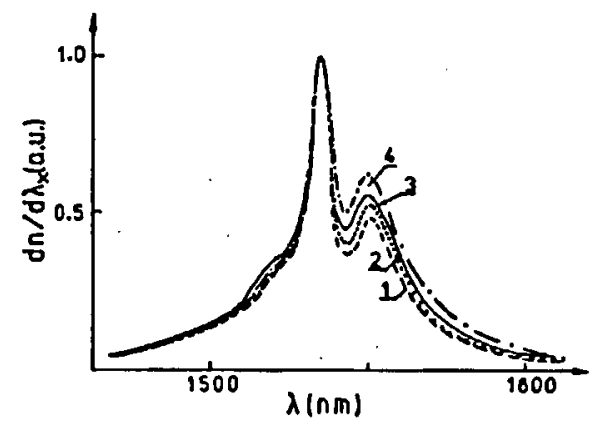

Fig. 2. Luminescence spectra of the Er-doped silica sol-gel glasses treated in freon atmosphere before vitrification (traces 1-3), measured at $\lambda_{e x}=520 \mathrm{~nm}$, and of the non-treated sample (trace 4 ).

demonstrate a decrease in intensity comparing to sample 4 . Since it has been shown [5] that fluorine influences significantly the chemistry of the sol-gel process, such changes of the luminescence profile might be associated with changes of the first coordination sphere of the erbium ion in the gel matrix.

The practical conclusion which can be drawn from this paper is that the freon heated treatment is a practical method of dehydration of the Er-doped silica sol-gel glasses, which significantly improves their optical quality. Moreover, due to this treatment the fluorine ions might significantly modify the nearest surroundings of the $\mathrm{Er}^{3+}$ ions.

\section{References}

[1] W.J. Miniscalo, in: Rare Earlh Doped Fiber Lasers and Amplifiers, Ed. M.J.F. Digonnct, Marcel Dekker, New York 1993, p. 19.

[2] R. Reisfeld, C.K. Joergensen, Struct. Bond. Vol. 77, (1992).

[3] E.N. Poddenezhny, I.M. Melnichenko, B.V. Plusch, I.Yu. Neroda, G.E. Malashkevich, J. Adv. Maller 5,1996 , in press; A.A. Boiko, E.N. Poddenezliny, J. Legendziewicz, J. Sokolnicki, E. Lukowiak, W. Stręk, J. Appl. Spectrosc. 62, 18 (1995).

[4] A.R. Silin, A.N. Truhin, Point Defects and Elementary Excitation in Crystal and Vilreous $\mathrm{SiO}_{2}$, Zinatne, Riga 1985, p. 244 (in Russian).

[5] C.J. Brinker, G.W. Sclierer, Sol-Gel Science, Academic Press, New York 1990. 